\title{
Et si le Brexit n'était que le début?
}

\section{Remo Osterwalder}

Dr, vice-président de la FMH, responsable du département Prestations et développement professionnel

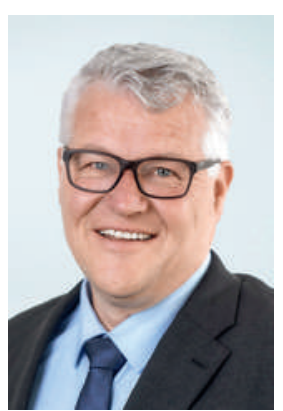

Non, je n'ai pas de boule de cristal et je ne peux pas non plus prédire précisément les conséquences du retrait de la Grande-Bretagne de l'Union européenne sur notre système de santé. Mais certains indices de nature économique permettent d'envisager plusieurs scénarios. Dans cet éditorial, je m'intéresserai à la question du personnel, des coûts et de l'économie.

Pour que son système de santé fonctionne, le RoyaumeUni, tout comme la Suisse, doit importer un grand nombre de médecins étrangers (35,4\%). Dans ce contexte, un système de santé étatisé (NHS) présente des inconvénients: la marge de manœuvre pour renforcer l'attrait de la profession médicale y est moins grande que dans notre système relativement libéral, et la relève fait vite défaut. Des parallèles existent cependant entre les deux systèmes: comme en Suisse, la libre circulation des personnes pourrait être fortement limitée en Grande-Bretagne. Or restreindre la libre circulation représente un risque pour les systèmes de santé qui reposent essentiellement sur le personnel étranger et peut déboucher sur une pénurie de main d'œuvre notamment dans les hôpitaux. Pour l'instant, aucune solution alternative (pools de ressources humaines, offres de prestations coordonnées ou croissance saine des cliniques ambulatoires) ne semble se détacher dans notre pays. De manière générale, les systèmes de santé réagissent plutôt lentement aux changements. Nous ferions donc bien de suivre avec attention la

Le Royaume-Uni recrute un très grand nombre de médecins étrangers. Or un système de santé étatique complique la recherche de personnel.

situation britannique en matière de recrutement du personnel pour voir si des solutions viables sont trouvées. Un scénario possible consisterait par exemple à lutter contre la déqualification des immigrés: pourquoi un médecin qualifié et bien formé ne peut-il pas pratiquer son métier uniquement faute d'accord sur la reconnaissance de la formation universitaire entre son pays d'accueil et son pays d'origine? Les hôpitaux pour- raient exploiter ce potentiel inutilisé à travers des programmes de formation complémentaire.

Le Royaume-Uni comme la Suisse profitent d'un accès facilité à Horizon 2020 et à d'autres programmes européens d'encouragement de la recherche et de l'innovation. Difficile de dire si le Brexit aura des conséquences sérieuses dans ce domaine; le transfert de connaissances est dans l'intérêt de tous et ne sera certainement pas remis en cause aussi facilement. En outre, la Grande-Bretagne dispose d'un net avantage grâce à ses contacts avec l'Inde et les Etats-Unis.

\section{Le Brexit renchérit les importations.}

La Grande-Bretagne devra-t-elle réduire l'offre médicale ou augmenter les impôts?

En revanche, les conséquences du Brexit sur l'évolution du cours de la livre sterling sont plus problématiques. Avec une devise britannique en chute de $10 \%$, l'achat de produits médicaux est devenu nettement plus cher. En 2015, la Grande-Bretagne a importé pour 33,1 milliards de produits pharmaceutiques, ce qui représente $5,3 \%$ de l'ensemble des importations, et pour 18,2 milliards de dispositifs médico-techniques, soit 2,9\% des importations; deux tiers des importations provenant de l'espace européen. Même avec une économie en relativement bonne santé, le Royaume-Uni pourra difficilement faire face à un renchérissement du coût des importations à hauteur de 2,7 milliards. Devra-t-il réduire l'offre médicale ou augmenter les impôts? Seul l'avenir le dira. Si à court terme, le retrait d'autres pays de l'Union européenne et un affaiblissement de l'Euro auraient des effets positifs pour le système de santé britannique, cela pourrait à l'inverse s'avérer catastrophique pour le pôle suisse de recherche et de production. En tant que pays exportateur, nous serions soumis à une pression considérable.

Nous serions donc bien inspirés de suivre l'évolution de la situation britannique en espérant que l'histoire européenne aille dans la bonne direction. 